\title{
First-principles calculations of Seebeck coefficients in a magnetic semiconductor $\mathrm{CuFeS}_{2}$
}

Hirokazu Takaki, Kazuaki Kobayashi, Masato Shimono, Nobuhiko Kobayashi, Kenji Hirose, Naohito Tsujii, and Takao Mori

Citation: Appl. Phys. Lett. 110, 072107 (2017); doi: 10.1063/1.4976574

View online: http://dx.doi.org/10.1063/1.4976574

View Table of Contents: http://aip.scitation.org/toc/apl/110/7

Published by the American Institute of Physics

\section{Articles you may be interested in}

Ferroelectric, pyroelectric, and piezoelectric properties of a photovoltaic perovskite oxide

Appl. Phys. Lett. 110, 063903063903 (2017); 10.1063/1.4974735

Electrically tunable diffraction efficiency from gratings in Al-doped $\mathrm{ZnO}$

Appl. Phys. Lett. 110, 071110071110 (2017); 10.1063/1.4976573

Spin-photo-currents generated by femtosecond laser pulses in a ferrimagnetic GdFeCo/Pt bilayer Appl. Phys. Lett. 110, 072402072402 (2017); 10.1063/1.4976202

Voltage induced mechanical/spin wave propagation over long distances

Appl. Phys. Lett. 110, 072401072401 (2017); 10.1063/1.4975828

Giant interfacial perpendicular magnetic anisotropy in $\mathrm{MgO} / \mathrm{CoFe} /$ capping layer structures

Appl. Phys. Lett. 110, 072403072403 (2017); 10.1063/1.4976517

High Curie temperature Mn5Ge3 thin films produced by non-diffusive reaction

Appl. Phys. Lett. 110, 072408072408 (2017); 10.1063/1.4976576
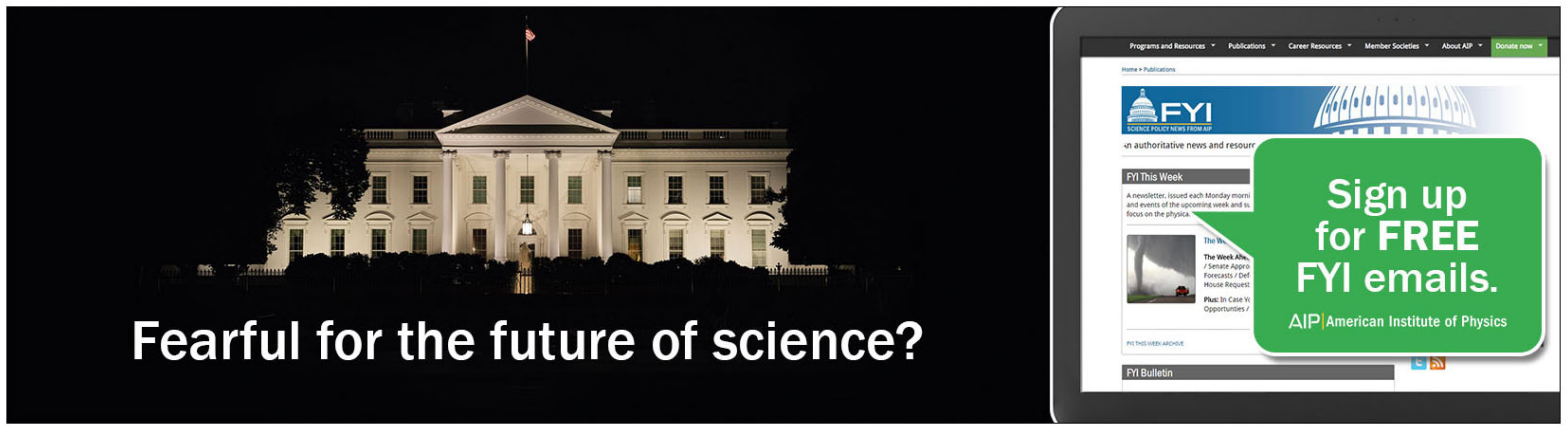


\title{
First-principles calculations of Seebeck coefficients in a magnetic semiconductor $\mathrm{CuFeS}_{2}$
}

\author{
Hirokazu Takaki, ${ }^{1,2, a)}$ Kazuaki Kobayashi, ${ }^{3}$ Masato Shimono, ${ }^{4}$ Nobuhiko Kobayashi, ${ }^{1}$ \\ Kenji Hirose, ${ }^{5}$ Naohito Tsujii, ${ }^{3}$ and Takao Mori ${ }^{3}$ \\ ${ }^{1}$ Faculty of Pure and Applied Sciences, University of Tsukuba, Tsukuba, Ibaraki 305-8573, Japan \\ ${ }^{2}$ National Institute for Materials Science (NIMS), Tsukuba, Ibaraki 305-0044, Japan \\ ${ }^{3}$ International Center for Materials Nanoarchitechtonics (MANA), National Institute for Materials Science \\ (NIMS), Tsukuba, Ibaraki 305-0044, Japan \\ ${ }^{4}$ Research Center for Structural Materials (RCSM), National Institute for Materials Science (NIMS), Tsukuba, \\ Ibaraki 305-0047, Japan \\ ${ }^{5}$ Smart Energy Research Laboratories, NEC Corporation, Tsukuba, Ibaraki 305-8501, Japan
}

(Received 10 November 2016; accepted 2 February 2017; published online 16 February 2017)

\begin{abstract}
We analyze the Seebeck coefficients of a magnetic semiconductor $\mathrm{CuFeS}_{2}$ using first-principles calculation methods based on density functional theory. The calculated temperature dependence of the Seebeck coefficient in the antiferromagnetic phase reproduces a distinctive behavior in a bulk $\mathrm{CuFeS}_{2}$, such as a peak structure at a low temperature and weak temperature dependence around room temperature. In doped systems, almost linear temperature dependence appears. Despite not including any effect beyond the conventional spin density functional theory in our calculations, the calculated results agree qualitatively with the experimental results. These agreements indicate that the behavior of the Seebeck coefficients in $\mathrm{CuFeS}_{2}$ is mainly determined by its electronic structure.
\end{abstract} Published by AIP Publishing. [http://dx.doi.org/10.1063/1.4976574]

Highly efficient thermoelectric materials have been attracting much attention because of their potential applications, especially for energy harvesting by waste heat. ${ }^{1-9} \mathrm{~A}$ challenge is to improve the relatively low conversion efficiency, which is a function of the figure of merit $Z T=\sigma S^{2} T / \kappa$, where $T$ is the temperature, $S$ is the Seebeck coefficient, $\sigma$ is the electrical conductivity, and $\kappa$ is the thermal conductivity, respectively. The numerator $\sigma S^{2}$ is the power factor. It is difficult to simply enhance $Z T$ because of the typical trade-off between the Seebeck coefficient and electrical conductivity, and the material that should conduct electricity well, without conducting heat, is also somewhat paradoxical.

Recently, magnetic semiconductors have been of great interest as a route to achieve a high power factor. $\mathrm{CuFeS}_{2}$, known as chalcopyrite, is a natural magnetic semiconductor, and one of the most intensively studied materials. ${ }^{10-16}$ $\mathrm{CuFeS}_{2}$, which is a natural compound, has a large Seebeck coefficient of approximately $500 \mu \mathrm{V} / \mathrm{K} .{ }^{10,11,13}$ Unfortunately, the bulk $\mathrm{CuFeS}_{2}$ does not have a small thermal conductivity and also a large electrical conductivity, because of which the figure of merit remains small. However, experimental results show that tuning the composition ratio of $\mathrm{Cu}, \mathrm{Fe}$, and $\mathrm{S}$ atoms in $\mathrm{CuFeS}_{2}$ by doping enhances the power factor drastically, up to approximately $1.0 \times 10^{-3} \mathrm{~W} \mathrm{~K}^{-2} \mathrm{~m}^{-1}$; therefore, a detailed understanding is necessary for the achievement of highly efficient thermoelectric materials. Electronic structures of $\mathrm{CuFeS}_{2}$ were analyzed in theoretical studies. ${ }^{17-19}$ These studies revealed that the antiferromagnetic phase stabilizes $\mathrm{CuFeS}_{2}$ compared to other magnetic phases, such as the para- and ferromagnetic phases. However, there are no theoretically calculated results for the thermoelectric properties of $\mathrm{CuFeS}_{2}$, such as the Seebeck coefficient. In particular,

${ }^{a)}$ Electronic mail: hrtakaki@bk.tsukuba.ac.jp the temperature dependence of the Seebeck coefficient in $\mathrm{CuFeS}_{2}{ }^{10,11}$ is an unsolved problem.

In this study, we analyze the Seebeck coefficient in $\mathrm{CuFeS}_{2}$ by the first-principles electronic structure calculation methods. The calculated Seebeck coefficient of $\mathrm{CuFeS}_{2}$ has a larger value compared to the value of typical nonmagnetic materials and is comparable to the previously reported experimental result. The calculated temperature dependence of the Seebeck coefficients qualitatively agrees with the experimental results. In the bulk system, the calculated result exhibits a peak in the low-temperature region and weak temperature dependence in the temperature region above the peak. In the doped systems, the Seebeck coefficients behave linearly with temperature. These agreements in the results between the conventional spin density functional theory and the experiments indicate that the behavior of the Seebeck coefficients in $\mathrm{CuFeS}_{2}$ is mainly determined by its electronic structure.

We employ the first-principles electronic structure calculation methods based on the density functional theory $(\mathrm{DFT})^{20,21}$ in this study. The unit cell of $\mathrm{CuFeS}_{2}$ is schematically shown in Fig. 1 . $\mathrm{CuFeS}_{2}$ has 16 atoms per unit cell, 4 atoms each of $\mathrm{Cu}$ and $\mathrm{Fe}$, and $8 \mathrm{~S}$ atoms. The unit cell and atomic positions are optimized by the plain-wave basis method implemented in Quantum Espresso code. ${ }^{22}$ For the comparison, we calculate three different magnetic phases, para-, ferro-, and antiferro-. For the calculations of the Seebeck coefficients, our first-principles electronic structure calculation code $\mathrm{SAKE}^{23,24}$ is employed. SAKE code is based on the DFT combined with the nonequilibrium Green's function (NEGF) formalism, which is called the DFT-NEGF method, ${ }^{25}$ with the atomically localized basis set. The atomic pseudopotentials and the atomically localized basis functions are generated by the adpack code. ${ }^{26}$ From the DFT-NEGF calculations, the transmission function $\tau(\varepsilon)$ between the left 


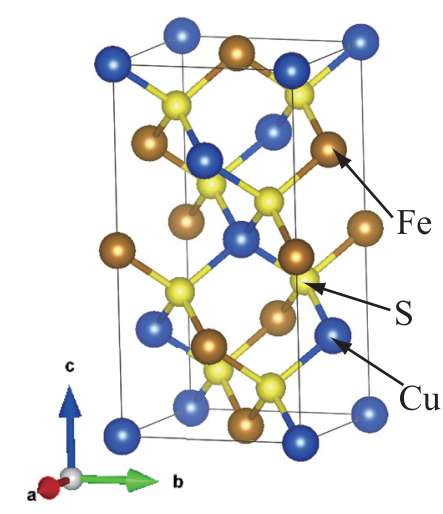

FIG. 1. Schematic view of the unit cell of $\mathrm{CuFeS}_{2}$. Blue, brown, and yellow balls represent $\mathrm{Cu}, \mathrm{Fe}$, and $\mathrm{S}$ atoms, respectively. The atomic positions and unit cells for three different magnetic phases, para-, ferro-, and antiferro-, are optimized by the plain-wave basis methods implemented in the Quantum Espresso code. ${ }^{22}$

and right ends of the unit cell as a function of the electron energy $\varepsilon$ is obtained. With this $\tau(\varepsilon)$, the Seebeck coefficient $S$ is written $\mathrm{as}^{24}$

$$
\begin{aligned}
S & =-(1 / e T) L_{1} / L_{0}, \\
L_{n} & =\frac{2}{h} \int_{-\infty}^{\infty} \mathrm{d} \varepsilon(\varepsilon-\mu)^{n}\left(-\frac{\partial f_{\mathrm{FD}}}{\partial \varepsilon}\right) \tau(\varepsilon),
\end{aligned}
$$

where $e, T, \mu$, and $f_{\mathrm{FD}}$ are the elementary charge, temperature, chemical potential, and Fermi-Dirac distribution function, respectively. In this work, we neglect the temperature dependence of the chemical potential. The transmission function $\tau(\varepsilon)$ is calculated under the zero-temperature situation, and the temperature dependence is generated via the Fermi-Dirac distribution function $f_{\mathrm{FD}}$.

We show the density of states of bulk $\mathrm{CuFeS}_{2}$ in three different magnetic phases, para-, ferro-, and antiferro-, and the transmission functions in Fig. 2. For the calculation of self-consistent electronic states by the plain-wave basis method, the the generalized gradiant approximation functional perametrized by Perdew, Burke, and Ernzerhof (GGA-PBE) functional, ${ }^{27}$ the cut-off energy for the wave functions (30 Ry), the electron density (300 Ry), and a $9 \times$ $9 \times 5 \mathrm{k}$ point mesh are used. In the para- and ferromagnetic phases, the systems are metallic; on the other hand, a narrow energy gap of approximately $0.05 \mathrm{eV}$ exists in the antiferromagnetic phase. This energy gap is about $10 \%$ of the experimentally reported values. ${ }^{28}$ However, other theoretical calculations based on the DFT with other types of the exchange-correlation functionals can overestimate the energy gap. ${ }^{19}$ In this work, we focus on the results based only on the GGA-PBE functional as the simplest but with a sufficient description of the electronic and magnetic structures of $\mathrm{CuFeS}_{2}$. The most stable phase is the antiferromagnetic phase, which is consistent with other theoretical and experimental results. The differences of the total energy in the para- and ferromagnetic phases relative to the antiferromagnetic phase are $\Delta E_{\text {para }}=E_{\text {para }}-E_{\text {antiferro }}=2.20 \mathrm{eV}$ and $\Delta E_{\text {ferro }}=E_{\text {ferro }}-E_{\text {antiferro }}=1.339 \mathrm{eV}, \quad$ respectively. The schemata of the ferro- and antiferromagnetic phases of $\mathrm{CuFeS}_{2}$ are also shown in the insets of Figs. 2(b) and 2(c). In
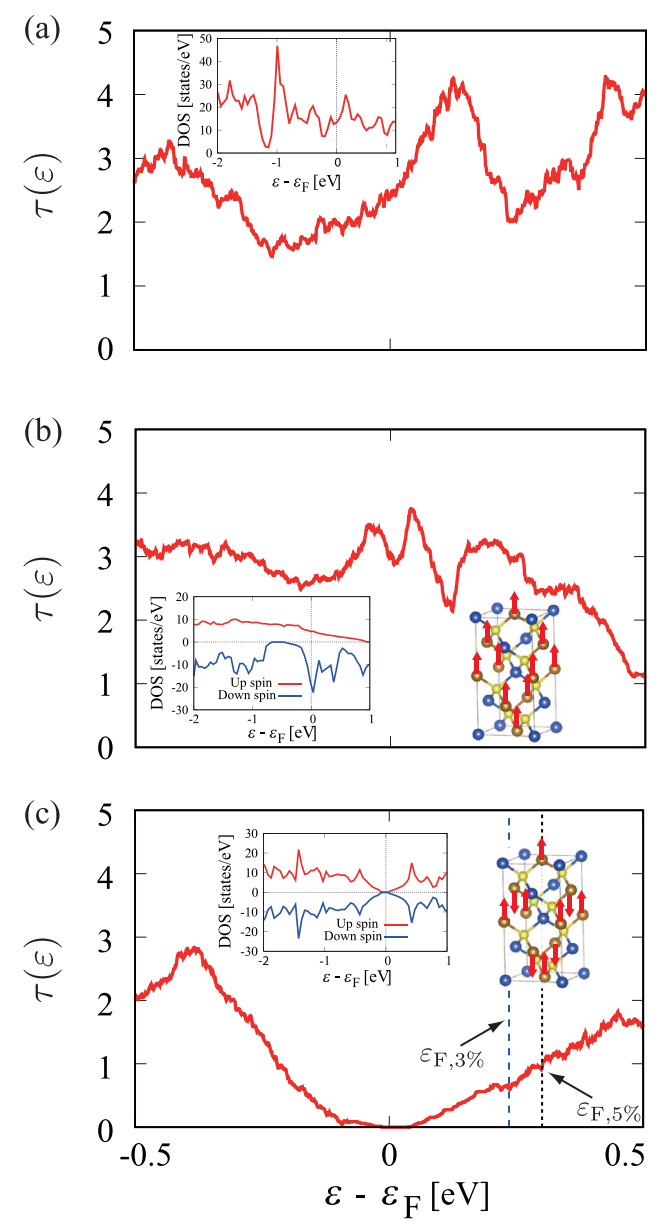

FIG. 2. The transmission functions of $\mathrm{CuFeS}_{2}$ around the Fermi level with three the different magnetic phases: (a) para-, (b) ferro-, and (c) antiferro-. The density of states is also shown in the insets. The three different magnetic phases are achieved by three different initial spin electron configurations. The ferro- and antiferromagnetic phases of $\mathrm{CuFeS}_{2}$ are also schematically shown in the insets (b) and (c): red arrows represent the magnetic moments of $\mathrm{Fe}$ atoms. The equations $\varepsilon_{\mathrm{F}, 3 \%}=\varepsilon_{\mathrm{F}}+0.236[\mathrm{eV}]$ and $\varepsilon_{\mathrm{F}, 5 \%}=$ $\varepsilon_{\mathrm{F}}+0.301[\mathrm{eV}]$ indicate the Fermi levels in $3 \%$ and $5 \%$ carrier doped systems, respectively.

$\mathrm{CuFeS}_{2}$, only $\mathrm{Fe}$ atoms have finite magnetic moments; therefore, each magnetic moment distributes parallel to the neighboring $\mathrm{Fe}$ atoms in the ferromagnetic phase and antiparallel in the antiferromagnetic phases, respectively. Here, we note that $\mathrm{CuFeS}_{2}$ has no other magnetic moment distributions in the antiferromagnetic phase except for the form shown in the inset of Fig. 2(c). The other possible magnetic structures which are not treated in this work are discussed in Ref. 19.

In Figs. 3(a) and 3(b), the calculated Seebeck coefficients in these three different magnetic phases are shown. In the para- and ferromagnetic phases, the electronic states exhibit a metallic nature, which leads to small magnitudes of $S(T)$. On the other hand, $S(T)$ has a relatively large value in the antiferromagnetic phase shown in Fig. 3(c), because of the semiconducting electronic nature. For the enhancement of the Seebeck coefficient, the rapid change of the transmission function, the density of states, or the electrical conductivity in energy around the chemical potential plays a key role. The transmission functions in the three magnetic phases are shown in Fig. 2. These transmission functions are calculated with the $\mathrm{Cu}-\mathrm{s} 2 \mathrm{p} 2 \mathrm{~d} 2, \mathrm{Fe}-\mathrm{s} 2 \mathrm{p} 2 \mathrm{~d} 2$, and $\mathrm{S}$-s2p2d1 basis set, the GGA-PBE functional, ${ }^{27}$ the $21 \times 21 \mathrm{k}$ point mesh, 

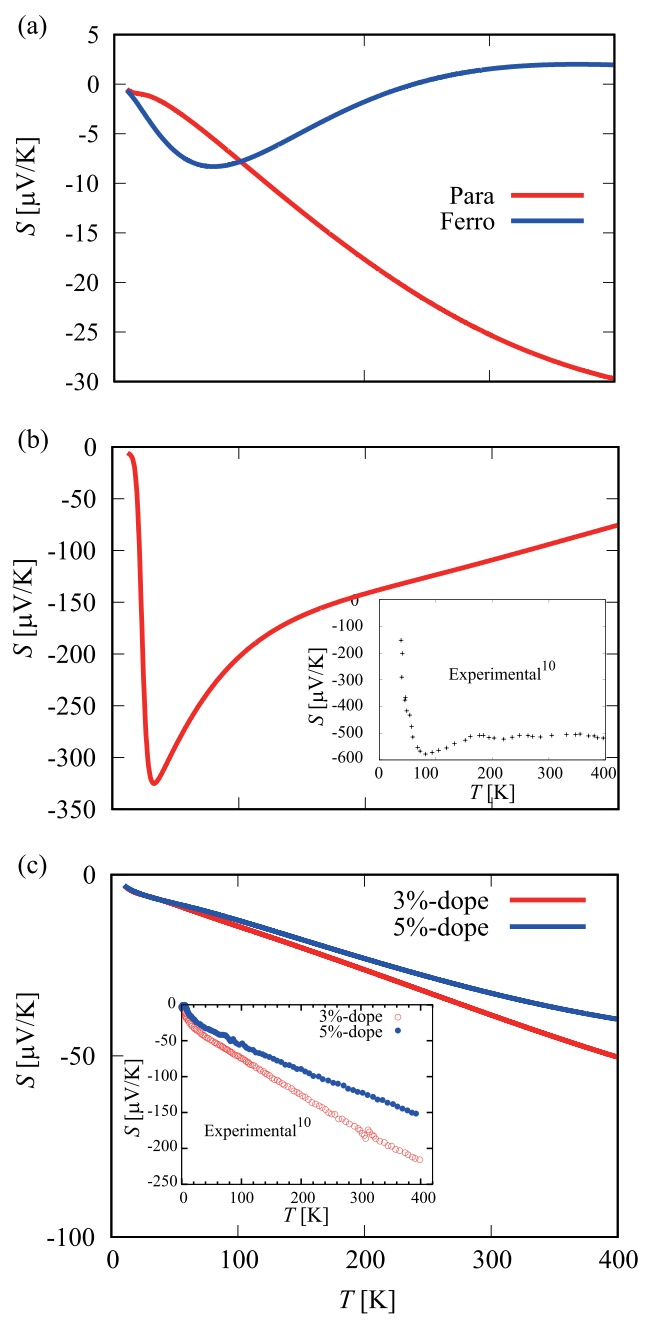

FIG. 3. The Seebeck coefficients of $\mathrm{CuFeS}_{2}$ in three different magnetic phases: (a) para- and ferro-, and (b) the antiferromagnetic phase, and (c) antiferromagnetic phase with $3 \%$ and $5 \%$ carrier doping, respectively. The systems in the para- and ferromagnetic phases are completely metallic which produces small values of the Seebeck coefficients. On the other hand, the system in the antiferromagnetic phase, which has a small energy gap, has a relatively large Seebeck coefficient. The doped system $S$ depends almost linearly on $T$. The experimental results ${ }^{10}$ in the bulk and doped $\mathrm{CuFeS}_{2}$ are also shown in the insets of (b) and (c). The calculated temperature dependences in the antiferromagnetic phase qualitatively agree well with the experimental results in both bulk and doped systems.

and the $0.5 \mathrm{meV}$ energy mesh. At the first sight, the $\tau(\varepsilon)$ in the para- and ferromagnetic phases have a strong dependence on $\varepsilon$. However, in the metallic systems, $\left(-\partial f_{\mathrm{FD}} / \partial \varepsilon\right)$ in Eq. (1) has a smoothing effect on $\tau(\varepsilon)$, which smears out the rapid change of $\tau(\varepsilon)$ with respect to $\varepsilon$. In the semiconducting system, on the other hand, this smoothing has little effect around the band edges, and then the semiconducting system has a large Seebeck coefficient compared to the metallic systems. The experimental result ${ }^{10}$ is also shown in the inset of Fig. 3(b). The calculated $S(T)$ exhibits a peak at a low temperature of approximately $20 \mathrm{~K}$, and weak temperature dependence after $100 \mathrm{~K}$. This behavior qualitatively agrees well with the experimental result, ${ }^{10}$ which demonstrates that $\mathrm{CuFeS}_{2}$ is a potentially good thermoelectric material.

We also calculate the Seebeck coefficients in doped $\mathrm{CuFeS}_{2}$ corresponding to the experimentally reported cases. Experimental studies showed that the temperature dependence of the Seebeck coefficient in $\mathrm{CuFeS}_{2}$ is drastically changed by the composition change. The Seebeck coefficients in two different compositions, $\mathrm{Cu}_{0.97} \mathrm{Fe}_{1.03} \mathrm{~S}_{2}$ and $\mathrm{Cu}_{0.95} \mathrm{Fe}_{1.05} \mathrm{~S}_{2}$, were reported. ${ }^{10}$ These two compositions, $\mathrm{Cu}_{0.97} \mathrm{Fe}_{1.03} \mathrm{~S}_{2}$ and $\mathrm{Cu}_{0.95} \mathrm{Fe}_{1.05} \mathrm{~S}_{2}$, correspond to $3 \%$ and $5 \%$ donor doped $\mathrm{CuFeS}_{2}$, respectively. The doping effect is treated here as the shift in the Fermi level, while the electronic structure shown in Fig. 2(c) remains unchanged. The magnitude of the Fermi level shift in each system is estimated by the condition $\Delta N=\int_{\varepsilon_{\mathrm{F}}}^{\varepsilon_{\mathrm{F}}+\Delta \varepsilon} \mathrm{d} \varepsilon D(\varepsilon), \Delta \varepsilon>0$. Here, $\Delta N, \Delta \varepsilon$, and $D(\varepsilon)$ are the number of doped electrons, Fermi level shift, and the density of states, respectively. The Fermi levels corresponding to the $3 \%$ and $5 \%$ doped systems are indicated in Fig. 2(c), and their amount of Fermi level shifts are $\Delta \varepsilon_{3 \%}=0.236[\mathrm{eV}]$ and $\Delta \varepsilon_{5 \%}=0.301[\mathrm{eV}]$, respectively. The Seebeck coefficients with $3 \%$ and $5 \%$ doped systems are shown in Fig. 3(c). With the shifts in the Fermi level, the doped systems are no longer semiconducting, but metallic. As a result, the magnitudes of these Seebeck coefficients are reduced from the bulk value and depend almost linearly on $T$. This $T$ linear dependence is a typical behavior in metallic systems explained by the Mott formula. ${ }^{29}$

The behavior of the Seebeck coefficients expressed by the present calculations can be explained briefly by the analytical form of $S(T)$. The integral $L_{n}$ in Eq. (1) has a finite value only when its integrand is even with respect to $\mu$. Thus, only the odd components contribute to the $L_{1}$, and only the even components to the $L_{0}$. As a result, the Seebeck coefficient can be expressed in an analytical form

$$
S(T)=\left(-\frac{1}{e}\right) \frac{\sum_{n=1} \alpha_{2 n} \tau_{2 n-1} T^{2 n-1}}{\sum_{n=0} \alpha_{2 n} \tau_{2 n} T^{2 n}},
$$

where $\tau_{n}$ denotes the expansion coefficients of $\tau(\varepsilon)$; i.e.

$$
\tau(\varepsilon)=\sum_{n=0}^{\infty} \tau_{n}(\varepsilon-\mu)^{n}, \quad \tau_{n}=\left.\frac{1}{n !} \frac{\mathrm{d}^{n} \tau}{\mathrm{d} \varepsilon^{n}}\right|_{\varepsilon=\mu} .
$$

The coefficient $\alpha_{2 n}$ is obtained with the formula: $\int_{0}^{\infty} \mathrm{d} x x^{n}\left[\left(e^{x}+1\right)\left(e^{-x}+1\right)\right]^{-1}=\left(1-2^{1-n}\right) \zeta(n) \Gamma(n)$, where $\zeta(n)$ and $\Gamma(n)$ are the Riemann zeta function and the gamma function, respectively. ${ }^{30}$ If $\tau(\varepsilon)$ is expanded up to the first order $\tau_{1}$, this analytical form recovers the Mott formula, ${ }^{29}$ in which $S(T)$ depends linearly on $T$. For the bulk $\mathrm{CuFeS}_{2}$, as one can see in the low-temperature region, the higher-order $\tau_{n}$ does not affect the $S(T)$, and hence, $S(T)$ behaves almost linearly up to the bottom of the peak. Above the temperature at the bottom of the peak, the higher-order $\tau_{n}$ makes a large contribution to $S(T)$. In the temperature region where the $L_{0}$ and $L_{1}$ make almost the same contribution in Eq. (1), the temperature dependence of $S(T)$ appears to be weak. For the doped $\mathrm{CuFeS}_{2}, \tau_{0}$ and $\tau_{1}$ are dominant components in $\tau(\varepsilon)$; thus, $S(T)$ has the form $\left(\tau_{1} / \tau_{0}\right) T$, which leads to $T$ linear behavior in the wide temperature region. In this analytical form, however, in the limit of $T \rightarrow \infty, S(T)$ definitely goes to zero. In addition, the Seebeck coefficients calculated here are also suppressed by the bipolar effect. In the narrow gap system, the integral of Eq. (1) for $n=1$ has two contributions from the conduction band $\varepsilon>\mu$ and the valence band $\varepsilon<\mu$. These two contributions have opposite signs, and thus, the 
contribution from the valance band reduces the magnitudes of the Seebeck coefficients in $\mathrm{CuFeS}_{2}$, which is an n-type thermoelectric material. The differences between the present calculated results and the experimental results should be explained by the neglected effects in this study; The temperature dependence of the chemical potential, the bipolar effect with tiny energy gap, and the interactions with other degrees of freedom, such as the magnon-drag effect, ${ }^{31}$ phonon-drag effect, ${ }^{32,33}$ and the spin fluctuation, ${ }^{34}$ whose contributions to $S(T)$ are proportional to $T^{3 / 2}, T^{3}$, and $T^{4}$, respectively.

To summarize, we carried out first-principles calculations based on the DFT to investigate the behavior and origins of the large Seebeck coefficient in $\mathrm{CuFeS}_{2}$. The results from the three different magnetic phases, para-, ferro-, and antiferro-, are compared. It is shown that the antiferromagnetic phase, where the magnetic moments in $\mathrm{Fe}$ atoms are ordered antiparallel to the neighboring ones, plays a key role for a large value of the Seebeck coefficient. The calculated temperature dependence of the Seebeck coefficients in the antiferromagnetic phase qualitatively agrees with the experimental results, reproduction of the peak structure at low temperature, weak temperature dependence near room temperature in bulk, and the linear temperature dependence in the doped systems. These agreements confirm that $\mathrm{CuFeS}_{2}$ is as a potentially good thermoelectric material and that the electronic structure of $\mathrm{CuFeS}_{2}$ mainly determines the behavior of the Seebeck coefficient. However, we still observe a relatively large gap between the magnitudes of the calculated and experimentally observed Seebeck coefficients. This indicates that further effects may be playing a key role, such as the temperature dependence of the chemical potential, precise estimation of the band gap, magnon-drag effect, phonon-drag effect, and spin fluctuation.

This work was supported by CREST, the Japan Science and Technology Agency. H.T. thanks Dr. I. Ohkubo of NIMS for the helpful information and discussions. T.M. and K.K. also acknowledge support from MANA Fusion. The numerical calculations were performed with Numerical Materials Simulator (SGI) in NIMS and the supercomputer systems B in the ISSP, University of Tokyo. The authors would like to thank Editage (www.editage.jp) for English language editing.

${ }^{1}$ L. E. Bell, Science 321, 1457 (2008).

${ }^{2}$ L. D. Hicks and M. S. Dresselhaus, Phys. Rev. B 47, 12727 (1993); 47, 16631 (1993).
${ }^{3}$ C. Wood, Rep. Prog. Phys. 51, 459 (1988).

${ }^{4}$ K. Biswas, J. He, I. D. Blum, C.-I. Wu, T. P. Hogan, D. N. Seidman, V. P. Dravid, and M. G. Kanatzidis, Nature 489, 414 (2012).

${ }^{5}$ Thermoelectric Nanomaterials, Materials Design and Applications, edited by K. Koumoto and T. Mori (Springer, 2013).

${ }^{6}$ Modules, Systems, and Applications in Thermoelectrics, edited by D. M. Rowe (CRC Press, 2012).

${ }^{7}$ Thermoelectrics, Basic Principles, and New Materials Development, edited by G. S. Nolas, J. Sharp, and J. Goldsmid (Springer, 2001).

${ }^{8}$ C. Dames, Scr. Mater. 111, 16 (2016).

${ }^{9}$ A. Maignan, E. Guilmeau, F. Gascoin, Y. Bréard, and V. Hardy, Sci. Technol. Adv. Mater. 13, 053003 (2012).

${ }^{10} \mathrm{~N}$. Tsujii and T. Mori, Appl. Phys. Express 6, 043001 (2013).

${ }^{11}$ N. Tsujii, T. Mori, and Y. Isoda, J. Electron. Mater. 43, 2371 (2014).

${ }^{12}$ R. Ang, A. U. Khan, N. Tsujii, K. Takai, R. Nakamura, and T. Mori, Angew. Chem. Int. Ed. 54, 12909 (2015).

${ }^{13}$ T. Teranishi, J. Phys. Soc. Jpn. 16, 1881 (1961).

${ }^{14}$ G. Donnay, L. M. Corliss, J. D. H. Donnay, N. Elliott, and J. M. Hastings, Phys. Rev. 112, 1917 (1958).

${ }^{15}$ Y. Li, T. Zhang, Y. Qin, T. Day, G. J. Snyder, X. Shi, and L. Chen, J. Appl. Phys. 116, 203705 (2014).

${ }^{16} \mathrm{~J}$. Li, Q. Tan, and J.-F. Li, J. Alloys Compd. 551, 143 (2013).

${ }^{17}$ T. Hamajima, T. Kambara, K. Gondaira, and T. Oguchi, Phys. Rev. B 24, 3349 (1981).

${ }^{18}$ M. Zhou, X. Gao, Y. Cheng, X. Chen, and L. Cai, Appl. Phys. A 118, 1145 (2015)

${ }^{19}$ S. Conejeros, P. Alemany, M. Llunell, I. de P. R. Moreira, V. Sańchez, and J. Llanos, Inorg. Chem. 54, 4840 (2015).

${ }^{20}$ P. Hohenberg and W. Kohn, Phys. Rev. 136, B864 (1964).

${ }^{21}$ W. Kohn and L. J. Sham, Phys. Rev. 140, A1133 (1965).

${ }^{22}$ P. Giannozzi, S. Baroni, N. Bonini, M. Calandra, R. Car, C. Cavazzoni, D. Ceresoli, G. L. Chiarotti, M. Cococcioni, I. Dabo, A. Dal Corso, S. de Gironcoli, S. Fabris, G. Fratesi, R. Gebauer, U. Gerstmann, C. Gougoussis, A. Kokalj, M. Lazzeri, L. Martin-Samos, N. Marzari, F. Mauri, R. Mazzarello, S. Paolini, A. Pasquarello, L. Paulatto, C. Sbraccia, S. Scandolo, G. Sclauzero, A. P. Seitsonen, A. Smogunov, P. Umari, and R. M. Wentzcovitch, J. Phys.: Condens. Matter 21, 395502 (2009).

${ }^{23}$ H. Takaki, N. Kobayashi, and K. Hirose, J. Nanomater. 2014, 172169.

${ }^{24}$ H. Takaki, K. Kobayashi, M. Shimono, N. Kobayashi, and K. Hirose, J. Appl. Phys. 119, 014302 (2016).

${ }^{25}$ M. Brandbyge, J.-L. Mozos, P. Ordejón, J. Taylor, and K. Stokbro, Phys. Rev. B 65, 165401 (2002).

${ }^{26}$ T. Ozaki and H. Kino, Phys. Rev. B 69, 195113 (2004).

${ }^{27}$ J. P. Perdew, K. Burke, and M. Ernzerhof, Phys. Rev. Lett. 77, 3865 (1996); 78, 1396 (1997).

${ }^{28}$ C. I. Pearce, R. A. D. Pattrick, and D. J. Vaughan, Rev. Mineral. Geochem. 61, 127 (2006).

${ }^{29}$ M. Cutler and N. F. Mott, Phys. Rev. 181, 1336 (1969).

${ }^{30}$ N. W. Ashcroft and N. D. Mermin, Solid State Physics (Holt-Saunders, 1976).

${ }^{31}$ F. J. Blatt, D. J. Flood, V. Rowe, P. A. Schroeder, and J. E. Cox, Phys. Rev. Lett. 18, 395 (1967).

${ }^{32}$ C. Herring, Phys. Rev. 96, 1163 (1954).

${ }^{33}$ L. Weber and E. Gmelin, Appl. Phys. A 53, 136 (1991).

${ }^{34}$ T. Moriya, Spin Fluctuations in Itinerant Electron Magnetism (Springer, 1985). 